\title{
Peristaltic Transport of a Couple Stress Fluid : Some Applications to Hemodynamics
}

\author{
S. Maiti ${ }^{1 *}, \quad$ J.C.Misra ${ }^{2 \dagger}$ \\ ${ }^{1}$ School of Medical Science and Technology \& Center for Theoretical Studies, \\ IIT, Kharagpur-721302, India \\ ${ }^{2}$ Professor of Applied of Mathematics and Pro-Vice-chancellor (Academic), \\ SOA University, Bhubaneswar-751030, India \\ (Formerly, Professor and Chairman, Department of Mathematics, \\ IIT Kharagpur, India)
}

\begin{abstract}
The paper deals with a theoretical investigation of the peristaltic transport of a couple stress fluid in a porous channel. The study is motivated towards investigating the physiological flow of blood in the micro-circulatory system, by taking account of the particle size effect. The velocity, pressure gradient, stream function and frictional force of blood are investigated, when the Reynolds number is small and the wavelength is large, by using appropriate analytical and numerical methods. Effects of different physical parameters reflecting porosity, Darcy number, couple stress parameter as well as amplitude ratio on velocity profiles, pumping action and frictional force, streamlines pattern and trapping of blood are studied with particular emphasis. The computational results are presented in graphical form. The results are found to be in good agreement with those of Shapiro et. al [52] that was carried out for a non-porous channel without consideration of couple stress effect. The present study puts forward an important observation that for peristaltic transport of a couple stress fluid during free pumping, flow reversal can be considerably controlled by suitably adjusting the couple stress effect of the fluid/Darcy permeability of the channel. It is also possible to avoid the occurrence of trapping, by reducing the permeability.
\end{abstract}

*Email address: somnathm@cts.iitkgp.ernet.in (S. Maiti)

${ }^{\dagger}$ Email address: misrajc@rediffmail.com (J.C. Misra) 
Keywords: Peristaltic Transport; Couple Stress Fluid; Porosity; Darcy number; Trapping.

\section{Introduction}

Peristalsis is a natural mechanism of pumping that is observed in the case of most physiological fluids. In the transport of some other fluids also peristaltic behaviour is observed. This behaviour is usually associated with a progressive wave of area contraction or expansion along the length of the boundary of a fluid-filled distensible tube. Peristaltic transport occurs in various physiological activities, for example, in the flow of urine from kidney to the bladder, in the movement of food material through the digestive tract, in flow of fluids through lymphatic vessels as well as in semen movement in the vas differences, in the movement of bile from gall bladder into the duodenum and spermatozoa inside the ductus efferentes of the male reproductive tract and cervical canal, in flow of ovum in the fallopian tube, the movement of cilia and also in the flow of blood through small blood vessels. This phenomenon is also applied in the propulsion of some industrial fluids. 


\begin{tabular}{|ll|}
\hline Nomenclature & \\
$a$ & Wave amplitudes \\
$D a$ & Half-width of the channel \\
$H$ & Darcy number \\
$k$ & Vertical displacement of the wall \\
$\alpha, \eta$ & Permeability parameter \\
$P$ & Couple stress parameters \\
$Q$ & Fluid pressure \\
$R$ & Flux at any axial station \\
$t$ & Reynolds number of the fluid \\
$X, Y$ & Time \\
$U, V$ & Rectangular Cartesian co-ordinates \\
$m_{1}, m_{2}$ & Velocity components in X,Y directions respectively \\
$\delta$ & Constants defined in equation (18) \\
$\Delta p$ & Wave number \\
$\epsilon$ & Pressure difference between the channel ends \\
$\lambda$ & Porosity parameter \\
$\mu$ & Wave length of the traveling wave motion of the wall \\
$\nu$ & Viscosity of the fluid \\
$\phi$ & Kinematic viscosity of the fluid \\
$\rho$ & Amplitude ratio \\
\hline
\end{tabular}

This mechanism is also used in many biomedical appliances, such as finger pumps, heart-lung machine, blood pump machine, dialysis machine and also in industries for the transport of noxious fluid in nuclear industries, as well as in roller pumps. For this reason in current years, studies of peristaltic movement have been receiving growing interest of scientific researchers. Much of the early literature of theoretical investigations, arranged according to the geometry, the type of fluids, the Reynolds number, wave amplitude, wavelength and the wave shape, along with an account of experimental studies on peristaltic transport have been reviewed by Srivastava and Srivastava [1]. Some of the important theoretical studies on peristalsis have been discussed by Eytan et al. [2], Jimenez-Lozano et al. [3], Misra et al [4, 5, 6, 7, 8, 9, 10, 11], Maiti and Misra [12], Usha and Rao [13], Mishra and Rao [14], Rao and Mishra [15], Park et al 
[16] as well as by Akbar and Nadeem[17]. A few of these studies have been carried out by using the lubrication theory by neglecting the fluid inertia and wall curvature without any restriction of the wave amplitude. Some other analyses are based on the consideration of small peristaltic wave amplitude, where the Reynolds number is arbitrary. Antanovskii and Ramkissoon [18] studied the peristaltic transport of a compressible viscous fluid through a pipe with the help of lubrication theory when the pressure drop changes with time, by taking into account the wall deformation of the pipe.

Bergel [19] observed that the capillary walls are surrounded by flattened endothelial cell layers which are porous. Dash et al. [20] considered the Brinkman equation to model blood flow in a coronary artery in the pathological state when clogging of blood occurs in the lumen of the artery. They assumed the clogged region as a porous medium and assumed the permeability to be constant/varying in the radial direction. Misra et al. [21, 22, 23, 24, 25, 26] studied different aspects of blood flow in arteries in normal/pathological states. Some models on blood rheology have been discussed by Chien and Skalak (cf. [28, 29]).

It is well known that most physiological fluids including blood behave as a non-Newtonian fluid. It has been suggested that blood flow behaviour in small vessels (of diameter less than $0.2 \mathrm{~mm}$ ) at low shear rate $\left(<20 \mathrm{sec}^{-1}\right)$ can be represented by a power law fluid [30, 31]. It was reported by Blair and Spanner [32] that blood can be represented by Casson's model for moderate shear rate flows. The non-Newtonian behaviour of blood is mainly due to the suspension of red blood cells in the plasma. When neutrally buoyant corpuscles are contained in a fluid and there exists a velocity gradient due to shearing stress, corpuscles have rotatory motion. Furthermore, it is observed that corpuscles have spin angular momentum, in addition to orbital angular momentum. As a result, the symmetry of stress tensor is lost in the fluid motion that is subjected to spin angular momentum. The fluid that has neutrally buoyant corpuscles, when observed macroscopically, exhibits non-Newtonian behaviour, and its constitutive equation is expressed by a stress tensor[41]. The radius of gyration of the corpuscles for such fluids is different from that of the fluid particles. Their difference produces couple stress in the fluid. It is known that such a fluid has spin angular momentum in addition to the couple stress effect. The importance of consideration of couple stress effects in studies of physiological and some other fluids was indicated by Cowin [33] and Beg et al [34, 35].

Studies pertaining to the couple stress fluid behaviour are very useful, because such studies bear the potential to better explain the behaviour of rheologically complex fluids, such as liquid crystals, polymeric suspensions that has have long-chain molecules, lubrication as well 
as human/sub-human blood [41, 36, 37, 38]. Valanis and Sun [39] as well as Popel et al.[40] remarked that couple stress fluids constitute a special class of non-Newtonian fluids that take account of the particle size. The couple stress theory presented by Stokes [41] is quite suitable to study blood flow in micro-vessels by taking the size of the erythrocytes into account. Shehawey and Mekheimer [42] analysed the flow of a couple stress fluid for any arbitrary Reynolds number and wave number, considering the wave amplitude to be small.

It may be mentioned that consideration of porosity is very much necessary to properly explain the fluid dynamical process that occurs in different parts of the body, such as vascular beds, lungs, kidneys and tumorous vessels. The importance of consideration of Darcian drag effects in studies related to flow of blood and some other fluids through porous media has been discussed by different researchers [43, 44, 45, 46, 47, 48, 49, 50]. Moreover, in many biomechanical studies, porosity of the media has significant influence on the transport of fluids. This applies more particularly to vessels impeded by clots and also to highly perfused skeletal tissues, tumors and to soft connecting tissues [51]. Some further discussion on vessel porosity and peristaltic flows of physiological fluids is available in several scientific publications $[52,53,54,55,56,57,58]$.

Being motivated by the observations reported in the above mentioned studies, we have undertaken here a study that concerns peristaltic flow of blood in a porous bed. With an aim to take account of the particle size effect, blood has been modelled as a couple-stress fluid. The problem has been analysed by using lubrication theory [52]. The analysis and the results of the study are particularly applicable to the peristaltic transport of blood in coronary arteries of smaller dimensions in the pathological state, when the lumen of the segment of a small blood vessel turns into a porous medium due to the presence of numerous blood clots, or when arterial clogging takes place by deposition of fatty plaques of cholesterol in the arterial lumen, and also in cases where numerous tumors are grown inside the lumen due to excessive cell division.

The results of the present study will serve as a reasonably good estimate of various fluid mechanical parameters for peristaltic transport of blood in small blood vessels in a pathological state. Since flow behaviour in an axi-symmetric vessel resembles that in a channel, we have studied here two-dimensional channel flow of the fluid. The results of this study will be applicable to blood vessels in the micro-circulatory system without any restriction.

The study bears promise of multi-fold important applications. In the realm of physiological fluid dynamics, in many situations it is required to have estimates of a variety of fluid mechanical variables when some physiological fluid has to pass through porous structures, particularly in pathological states. The study will also have an important bearing in 
examining the flow in a vessel when the luminal surface of endothelial layer is attached with glycocalyx, which contains a series of micro-molecules and adsorbed plasma proteins. The present study can also find important applications in artificial bio-processors that have synthetic porous surfaces. Since blood flowing in small vessels has got relevance with flow in porous media, the study is applicable to blood flow in micro-vessels more particularly in situations where couple stress effects of blood are prominent. In addition to all these, the analysis presented here should find applications in polymer industries, where it is often required to examine the flow behaviour of different types of fluids passing through porous structures.

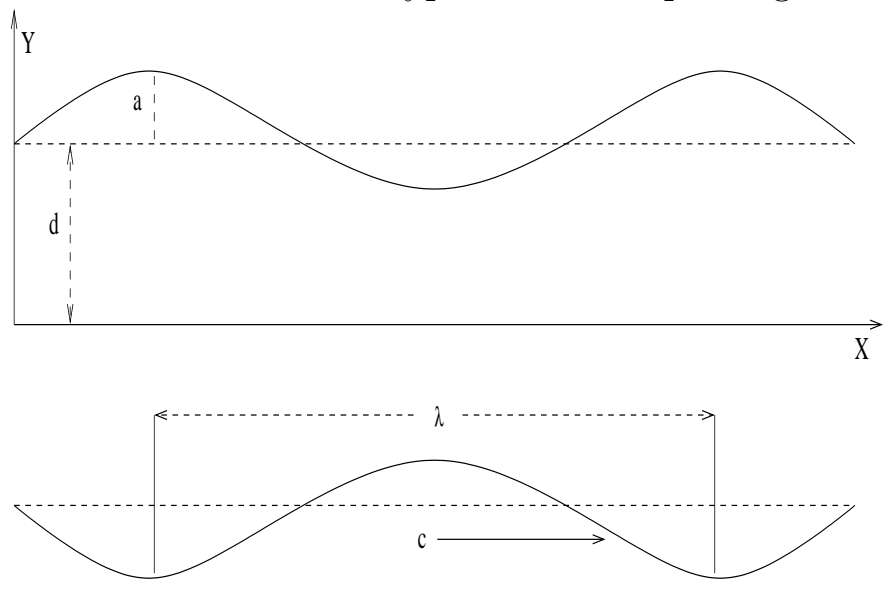

Fig. 1: A physical sketch of the problem

\section{Mathematical Modelling and Analysis}

Let us consider the peristaltic motion of blood on a porous channel. Blood is treated as a viscous couple stress fluid (non-Newtonian). We take (X,Y) as Cartesian coordinates of a point, $\mathrm{X}$ being measured in the direction of wave propagation and $\mathrm{Y}$ in the direction normal to the mean position of the corresponding small blood vessel. In the analysis that follows we consider channel flow. The constitutive equations and equations of motion for couple stress fluid flow in the absence of body moment and body couple can be put as [41, 36, 37, 38]

$$
\begin{gathered}
\tau_{j i, j}=\rho \frac{d v_{i}}{d t} \\
e_{i j k} T_{j k}^{A}+M_{j i, j}=0 \\
\tau_{i j}=-P \delta_{i j}+2 \mu d_{i j} \\
\mu_{i j}=4 \eta \omega_{j, i}+4 \eta^{\prime} \omega_{i, j}
\end{gathered}
$$


in which $\rho$ is the fluid density, $\tau_{i j}$ and $T_{i j}^{A}$ designate the symmetrical and antisymmetrical parts of the stress tensor $T_{i j}, v_{i}$ the velocity vector, $M_{i j}$ the couple stress tensor, $\mu_{i j}$ designate the deviatoric part of $M_{i j}, \omega_{i}$ stands for the vorticity vector, $d_{i j}$ is the symmetric part of the velocity gradient, $\eta$ and $\eta^{\prime}$ are constants associated with the couple stress effect, $\mathrm{P}$ the pressure.

Let $Y=H$ and $Y=-H$ be respectively the upper and lower boundaries of the channel (cf. Fig. 1). The motion is considered to be induced by a progressive sinusoidal wave train propagating with a constant speed $c$ along the channel wall, so that we have

$$
H=d+a \cos \left(\frac{2 \pi}{\lambda}(X-c t)\right)
$$

where $a$ is the wave amplitude, $\lambda$ the wave length and $d$ the half width of the channel.

We shall use Brinkman model for investigating the flow through the porous medium. Thus the equations that govern the flow of the couple stress fluid in the porous channel may be written in the form

$$
\begin{gathered}
\frac{\partial U}{\partial X}+\frac{\partial V}{\partial Y}=0 \\
\rho\left(\frac{\partial U}{\partial t}+U \frac{\partial U}{\partial X}+V \frac{\partial U}{\partial Y}\right)=-\frac{\partial P}{\partial X}+\frac{\mu}{\epsilon} \nabla^{2} U-\eta \nabla^{4} U-\frac{\mu}{k} U \\
\text { and } \rho\left(\frac{\partial V}{\partial t}+U \frac{\partial V}{\partial X}+V \frac{\partial V}{\partial Y}\right)=-\frac{\partial P}{\partial Y}+\frac{\mu}{\epsilon} \nabla^{2} V-\eta \nabla^{4} V-\frac{\mu}{k} V
\end{gathered}
$$

in which $\mathrm{k}$ is the permeability parameter, $\eta$ the couple stress parameter, $\epsilon$ stands for the porosity parameter and $\nabla^{2}=\frac{\partial^{2}}{\partial X^{2}}+\frac{\partial^{2}}{\partial Y^{2}}$.

Considering a wave frame $(x, y)$ that moves with a velocity $c$ away from the fixed frame $(X, Y)$, let us use the transformations

$$
x=X-c t, \quad y=Y, \quad u=U-c, \quad v=V, \quad p(x, y)=P(X, Y, t)
$$

in which $(u, v)$ and $(U, V)$ are the velocity components in the wave frame and the fixed frame respectively, $p$ and $P$ stand for pressure in wave and fixed frames of reference.

Therefore the equation governing the flow of the fluid which is steady in the wave frame of reference can be written in the form

$$
\begin{gathered}
\frac{\partial u}{\partial x}+\frac{\partial v}{\partial y}=0 \\
\rho\left(u \frac{\partial u}{\partial x}+v \frac{\partial u}{\partial y}\right)=-\frac{\partial p}{\partial x}+\frac{\mu}{\epsilon} \nabla^{2} u-\eta \nabla^{4} u-\frac{\mu}{k} u-\frac{\mu c}{k} \\
\rho\left(u \frac{\partial v}{\partial x}+v \frac{\partial v}{\partial y}\right)=-\frac{\partial p}{\partial y}+\frac{\mu}{\epsilon} \nabla^{2} v-\eta \nabla^{4} v-\frac{\mu}{k} v
\end{gathered}
$$


Let us now introduce the following non-dimensional variables:

$$
\begin{aligned}
& \bar{x}=\frac{x}{\lambda}, \quad \bar{y}=\frac{y}{d}, \quad \bar{u}=\frac{u}{c}, \quad \bar{v}=\frac{v}{c \delta}, \quad \delta=\frac{d}{\lambda}, \quad \bar{p}=\frac{d^{2} p}{\mu c \lambda}, \quad \bar{t}=\frac{c t}{\lambda}, \quad h=\frac{H}{d}, \quad \phi=\frac{a}{d}, \\
& \alpha^{2}=\frac{\eta / \mu}{d^{2}}=\frac{l^{2}}{d^{2}}, \quad R=\frac{\rho c d}{\mu}, \quad D a=\frac{k}{d^{2}} .
\end{aligned}
$$

Here $l^{2}$ is a material constant that has dimension of length square. It can be identified with a property that depends on the size of the fluid molecule (cf. Singh [59]). Dropping the bars over the symbols, the equations governing the steady flow of the fluid can be rewritten as

$$
\begin{gathered}
\delta \frac{\partial u}{\partial x}+\frac{\partial v}{\partial y}=0 \\
R \delta\left(u \frac{\partial u}{\partial x}+v \frac{\partial u}{\partial y}\right)=-\frac{\partial p}{\partial x}+\frac{1}{\epsilon} \nabla^{2} u-\alpha^{2} \nabla^{4} u-\frac{1}{D a} u-\frac{1}{D a} \\
R \delta^{3}\left(u \frac{\partial v}{\partial x}+v \frac{\partial v}{\partial y}\right)=-\frac{\partial p}{\partial y}+\frac{\delta^{2}}{\epsilon} \nabla^{2} v-\alpha^{2} \delta^{2} \nabla^{4} v-\frac{\delta^{2}}{D a} v \\
\text { in which } \nabla^{2} \equiv \delta^{2} \frac{\partial^{2}}{\partial x^{2}}+\frac{\partial^{2}}{\partial y^{2}}
\end{gathered}
$$

\section{Boundary Conditions:}

Keeping in view the physical conditions of the problem, the boundary conditions in the fixed frame may be put mathematically as

(i) $\quad U_{Y}=0, \quad U_{Y Y Y}=0$ at $Y=0$

(ii) $\quad U=0, V=\frac{\partial H}{\partial t},-\left(V_{X X}-U_{Y X}\right) H_{X}+\left(V_{X Y}-U_{Y Y}\right)=0$ at $Y=H$

In the wave frame, these boundary conditions in terms of the non-dimensional variables will be transformed to

$$
\begin{aligned}
& \text { (i) } u_{y}=0, \quad u_{y y y}=0 \text { at } y=0 \\
& \text { (ii) } \quad u=0, \quad v=\frac{\partial h}{\partial t}, \quad-\left(\delta^{4} v_{x x}-\delta^{2} u_{y x}\right) h_{x}+\left(\delta^{2} v_{x y}-u_{y y}\right)=0 \text { at } y=h
\end{aligned}
$$

Applying long wave length approximation $(\delta \ll 1)$, for small Reynolds number (cf. Shapiro et al [52]), the equations (14) and (15) reduce to

$$
0=-\frac{\partial p}{\partial x}+\frac{1}{\epsilon} \nabla_{1}^{2} u-\alpha^{2} \nabla_{1}^{4} u-\frac{1}{D a} u-\frac{1}{D a}
$$




$$
\begin{array}{r}
\text { and } 0=-\frac{\partial p}{\partial y}, \\
\text { where } \nabla_{1}^{2} \equiv \frac{\partial^{2}}{\partial y^{2}}
\end{array}
$$

Thus the pressure turns out to be a function of $\mathrm{x}$ only. The solution of equation (19) satisfying the boundary conditions(18) is found in the form

$$
u=\frac{D a m_{2}^{2} \cosh \left(m_{1} y\right)}{\left(m_{2}^{2}-m_{1}^{2}\right) \cosh \left(m_{1} h\right)} \frac{d p}{d x}-\frac{D a m_{1}^{2} \cosh \left(m_{2} y\right)}{\left(m_{2}^{2}-m_{1}^{2}\right) \cosh \left(m_{2} h\right)} \frac{d p}{d x}-D a \frac{d p}{d x}-1
$$

where

$$
-h \leq y \leq h, m_{1}^{2}=\frac{1-\sqrt{1-4 \epsilon^{2} \alpha^{2} / D a}}{2 \epsilon \alpha^{2}} \text { and } m_{2}^{2}=\frac{1+\sqrt{1-4 \epsilon^{2} \alpha^{2} / D a}}{2 \epsilon \alpha^{2}}
$$

The rate of volume flow ' $\mathrm{q}$ ' through each section is a constant (independent of both $\mathrm{x}$ and $\mathrm{t}$ ). It is given by

$$
q=\int_{0}^{h} u d y .
$$

Substituting (21) and performing the integration, we find

$$
q=\frac{D a m_{2}^{2} \tanh \left(m_{1} h\right)}{m_{1}\left(m_{2}^{2}-m_{1}^{2}\right)} \frac{d p}{d x}-\frac{D a m_{1}^{2} \tanh \left(m_{2} h\right)}{m_{2}\left(m_{2}^{2}-m_{1}^{2}\right)} \frac{d p}{d x}-D a h \frac{d p}{d x}-h
$$

Hence the flux at any axial station in the fixed frame is found to be given by

$$
Q=\int_{0}^{h}(u+1) d y=q+h,
$$

while the expression for the time-averaged volumetric flow rate over one period $T\left(=\frac{\lambda}{c}\right)$ of the peristaltic wave is obtained as

$$
\bar{Q}=\frac{1}{T} \int_{0}^{T} Q d t=\frac{1}{T} \int_{0}^{T}(q+h) d t=q+1 .
$$

The pressure gradient obtained from equation (23) can be expressed as

$$
\frac{d p}{d x}=\frac{(q+h) m_{1} m_{2}\left(m_{2}^{2}-m_{1}^{2}\right)}{D a m_{2}^{3} \tanh \left(m_{1} h\right)-D a m_{1}^{3} \tanh \left(m_{2} h\right)-D a h m_{1} m_{2}\left(m_{2}^{2}-m_{1}^{2}\right)} .
$$

The pressure rise per wavelength and the frictional force can be calculated by using the relations

$$
\Delta p=\int_{0}^{\lambda}\left(\frac{d p}{d x}\right) d x .
$$




$$
F=-\int_{0}^{\lambda} h\left(\frac{d p}{d x}\right) d x .
$$

Integrating over one wave length, we find

$$
\begin{gathered}
\Delta p=\frac{m_{1} m_{2}\left(m_{2}^{2}-m_{1}^{2}\right)}{D a} \bar{Q} I_{1}+\frac{m_{1} m_{2}\left(m_{2}^{2}-m_{1}^{2}\right)}{D a} I_{2} \\
F=\frac{m_{1} m_{2}\left(m_{2}^{2}-m_{1}^{2}\right)}{D a} \bar{Q} I_{3}+\frac{m_{1} m_{2}\left(m_{2}^{2}-m_{1}^{2}\right)}{D a} I_{4}
\end{gathered}
$$

where

$$
\begin{gathered}
I_{1}=\int_{0}^{1} \frac{d x}{m_{2}^{3} \tanh \left(m_{1} h\right)-m_{1}^{3} \tanh \left(m_{2} h\right)-h m_{1} m_{2}\left(m_{2}^{2}-m_{1}^{2}\right)} \\
I_{2}=\int_{0}^{1} \frac{(h-1) d x}{m_{2}^{3} \tanh \left(m_{1} h\right)-m_{1}^{3} \tanh \left(m_{2} h\right)-h m_{1} m_{2}\left(m_{2}^{2}-m_{1}^{2}\right)} \\
I_{3}=-\int_{0}^{1} \frac{h d x}{m_{2}^{3} \tanh \left(m_{1} h\right)-m_{1}^{3} \tanh \left(m_{2} h\right)-h m_{1} m_{2}\left(m_{2}^{2}-m_{1}^{2}\right)} \\
\text { and } I_{4}=-\int_{0}^{1} \frac{h(h-1) d x}{m_{2}^{3} \tanh \left(m_{1} h\right)-m_{1}^{3} \tanh \left(m_{2} h\right)-h m_{1} m_{2}\left(m_{2}^{2}-m_{1}^{2}\right)} .
\end{gathered}
$$

In the fixed frame of reference, the expression for the non-dimensional axial velocity reads

$$
\begin{aligned}
& U(X, Y, t)= \frac{m_{1} m_{2}(\bar{Q}+h-1)}{m_{2}^{3} \tanh \left(m_{1} h\right)-m_{1}^{3} \tanh \left(m_{2} h\right)-h m_{1} m_{2}\left(m_{2}^{2}-m_{1}^{2}\right)} \times \\
&\left(\frac{m_{2}^{2} \cosh \left(m_{1} Y\right)}{\cosh \left(m_{1} h\right)}-\frac{m_{1}^{2} \cosh \left(m_{2} Y\right)}{\cosh \left(m_{2} h\right)}+m_{1}^{2}-m_{2}^{2}\right) \\
& \text { with } h=1+\phi \cos [2 \pi(X-t)] .
\end{aligned}
$$

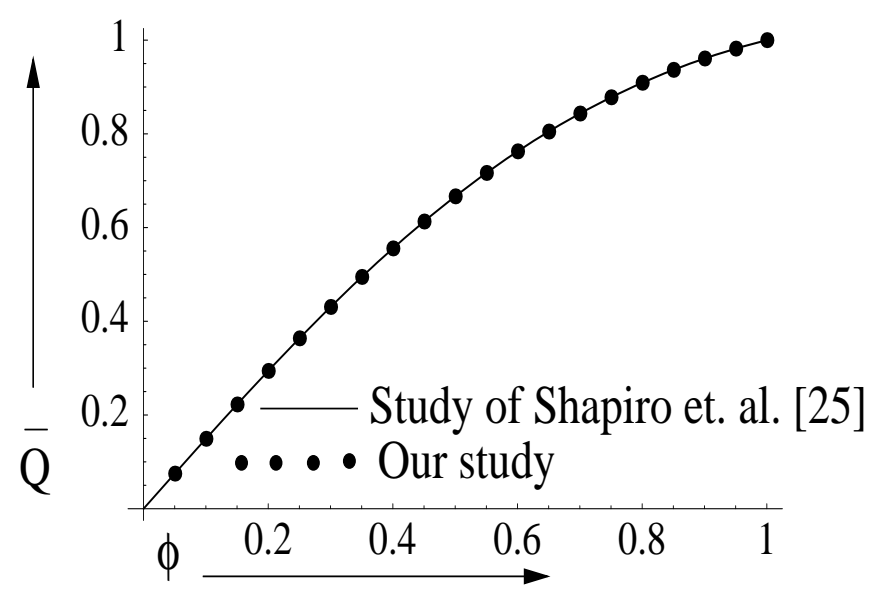

Fig. 2.1: Variation of $\bar{Q}$ with $\phi$ for $\Delta p=0, \alpha=0.001, \mathrm{Da}=10000$, e=0.9999. 


\section{Results and Discussion}

Analytical expressions of the pressure gradient, the volumetric flow rate, force of friction and axial velocity for the problem under consideration have been calculated and presented in the previous section. For the complexity of the problem, it was not possible to determine the analytical expression of $\Delta p$ and $\mathrm{F}$ in terms of $\bar{Q}$ and other parameters. Therefore, we had to employ appropriate numerical methods along with the use of the software mathematica. Similarly, an analytical treatment was found inadequate for finding the velocity as a function of $\Delta p, \mathrm{X}, \mathrm{Y}, \mathrm{t}$ and other related parameters. This necessitated calculating first

$$
\bar{Q}=\frac{D a \Delta p-m_{1} m_{2}\left(m_{2}^{2}-m_{1}^{2}\right) I_{2}}{m_{1} m_{2}\left(m_{2}^{2}-m_{1}^{2}\right) I_{1}} .
$$

For the computation of this also, we had to make use of numerical quadratures. In this section, the problem will be investigated further for a specific situation by employing appropriate numerical integration techniques with the help of mathematica software. For the quantitative study, the following ranges of values for different parameters involved in the model analysis have been considered :

$\phi=0.1$ to $0.9 ; \Delta p=-40$ to $40 ; \alpha=0.001$ to $1.0 ; \mathrm{Da}=0.05$ to 10000 , e=0.3 to 1.0, $\bar{Q}=-1$ to 3 (Cf. Refs. $[20,56,57,58]$ ).

\subsection{Pumping Characteristics}

Plots in Figs. 2 illustrate the variation of the volumetric flow rate of peristaltic waves with pressure gradient for different values of the amplitude ratio, couple stress parameter, permeability factor and porosity parameter. Fig.2.1 shows that the results computed on the basis of our study in the absence of permeability, porosity and couple stress parameters perfectly match with the results reported by Shapiro et al [52] for $\bar{Q}_{\max }$ when $\Delta p=0, \bar{Q}=Q / a c$. One may observe from Fig. 2.2 that in the range of values of the pressure gradient examined in the present study, the volumetric flow rate increases with the increase in the amplitude ratio in the entire pumping region $(\Delta p>0)$, in the free pumping zone $(\Delta p=0)$ as well as in the co-pumping region $(\Delta p<0)$ for $\Delta p>-2.0$. However, the trend reverses as soon as the pressure gradient drops below -2.0. These observations, of course, hold good for a particular set of values indicated in Fig. 2.2. Fig. 2.3 shows that in the entire pumping region the volumetric flow rate increases with the increase in couple stress parameter, whereas in the co-pumping region, a reverse trend is noticed. From the same figure, we further derive the information that for a Newtonian fluid 


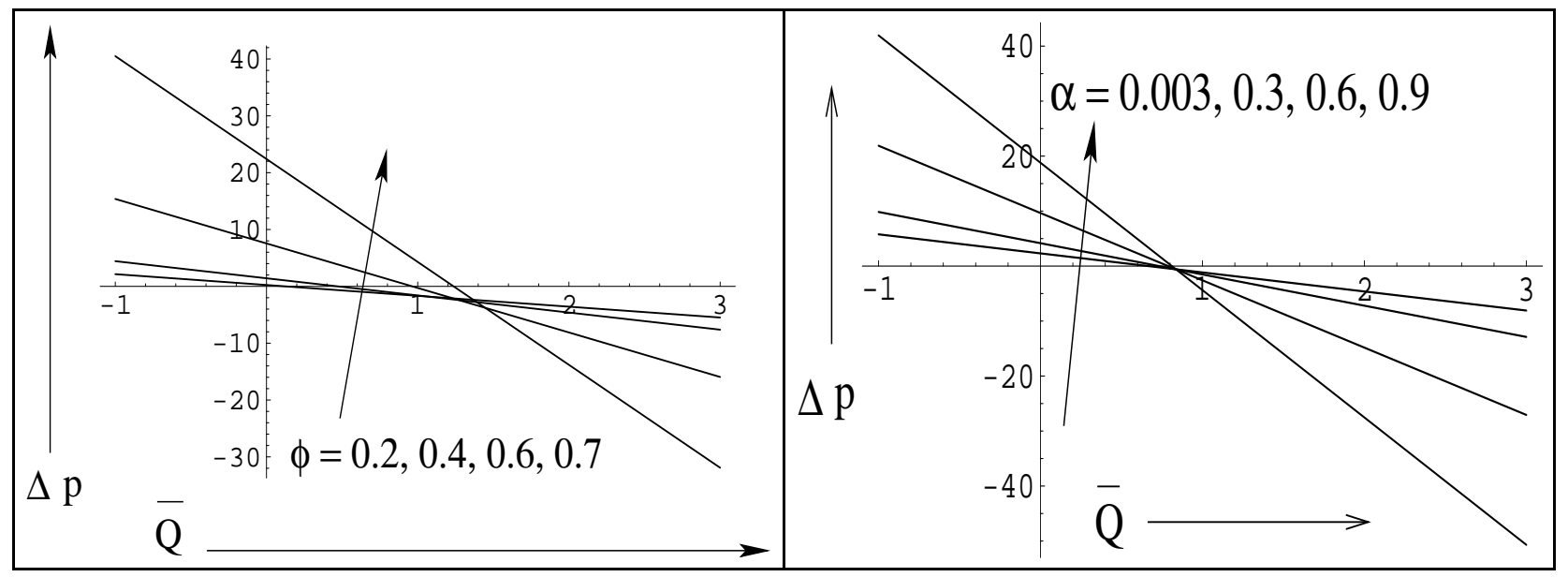

Fig. 2.2
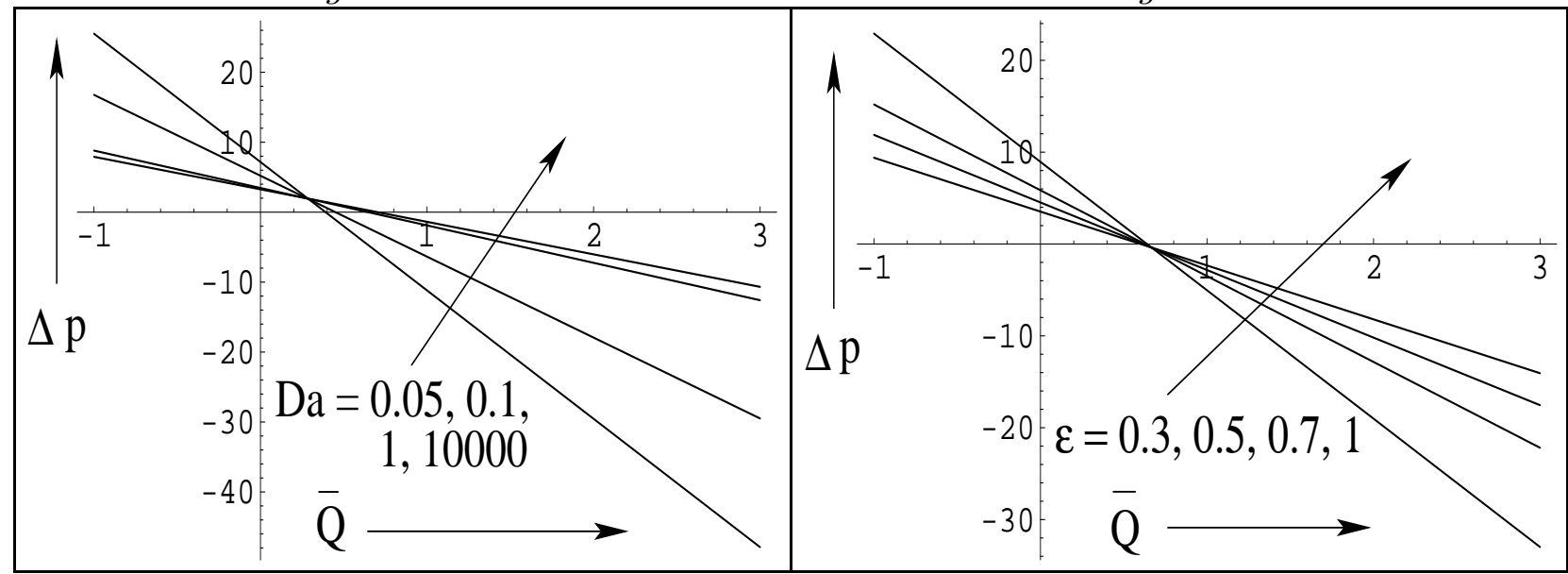

Fig. 2.4

Fig. 2.5

Fig 2.2-2.5: Variation of $\bar{Q}$ with $\Delta p$ (Fig 2.2) for different values of $\phi$ along with $\alpha=0.2, \mathrm{Da}=10000, \epsilon=1$; (Fig 2.3) for different values of $\alpha$ with $\phi=0.5, \mathrm{Da}=10000, \epsilon=1$; (Fig 2.4) for different values of Da with $\phi=0.5, \alpha=0.2, \epsilon=0.95$; (Fig 2.5) for different values of $\epsilon$ with $\phi=0.5, \alpha=0.2, \mathrm{Da}=0.5$ 


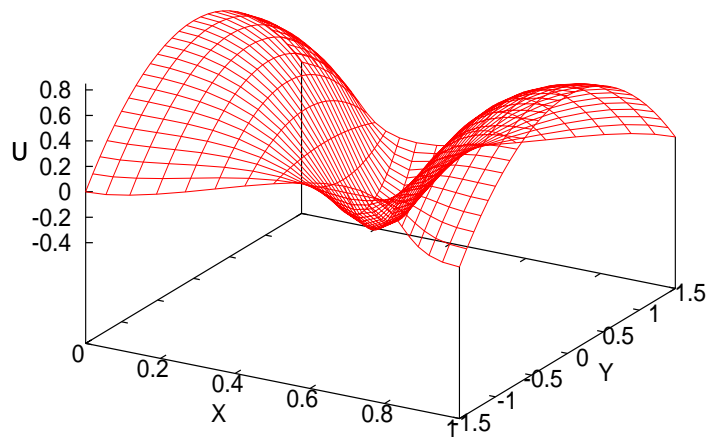

Fig. $3.1(t=0.0)$

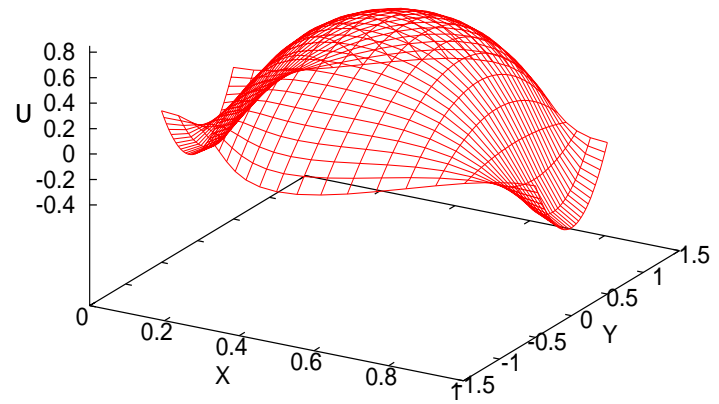

Fig. $3.3(t=0.5)$

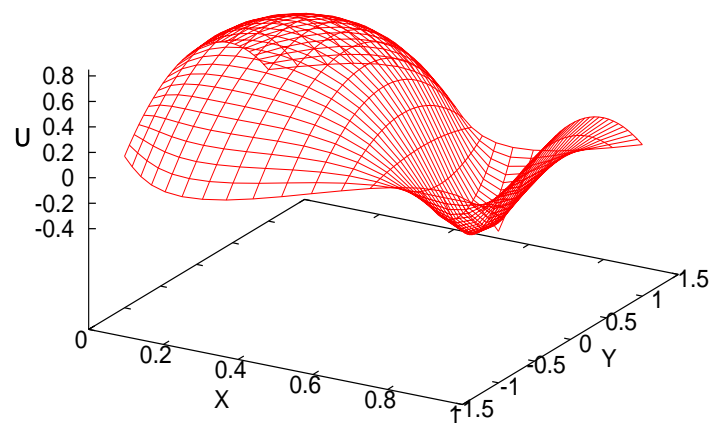

Fig. $3.2(t=0.25)$

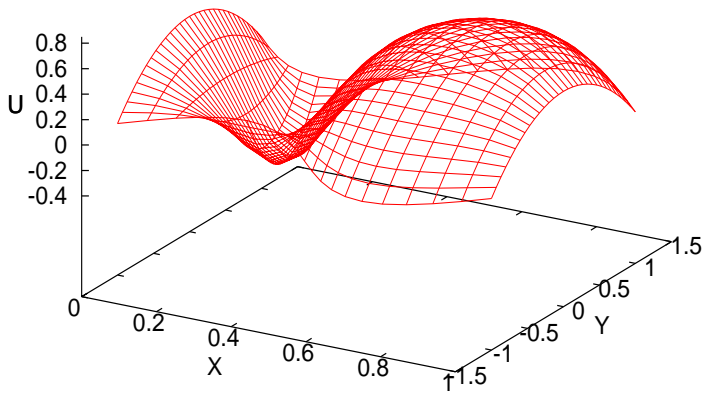

Fig. $3.4(t=0.75)$

Figs. 3.1-3.4: Aerial view of the velocity distribution at different times

$$
(\alpha=.003, D a=10000, \epsilon=1, \Delta p=0, \phi=.5)
$$


( $\alpha \simeq 0$ ), the magnitude of the pressure rise is less than that for the couple stress fluid both in pumping and co-pumping regions. One can observe from Fig. 2.4 that the volumetric flow rate can be gradually reduced in the pumping region when $\Delta p>5$ and enhanced in the pumping region when $\Delta p<5$, free pumping region as well as in the co-pumping region by increasing the Darcy number through some mechanism. It is found from Fig. 2.5 that with a rise in the porosity parameter, volumetric flow rate increases in the pumping region, but reduces in the co-pumping region.

\subsection{Velocity Change}

An idea of the distribution of axial velocity can be from Figs 3 in the cases of free pumping (Figs. 3.1-3.9), co-pumping (Fig. 3.10 and 3.12) and pumping (Fig. 3.11) zones for different values of the amplitude ratio, the couple stress parameter, the Darcy number and the porosity parameter. In the fixed frame of reference, since the velocity profiles and the height of the channel change with time, the axial velocity has been studied at the particular time instant $\mathrm{t}=\mathrm{T} / 4$. Aerial view of the velocity distribution of a Newtonian fluid on the basis of our study has been presented in Figs 3.1-3.4, while Fig. 3.5 gives the velocity distribution of a couple stress fluid $(\alpha=0.4)$ in the plane of the channel. We observe that at any instant of time there exists a retrograde flow region. But the forward flow region is predominant here as the time averaged flow rate $\bar{Q}$ is positive. Our study also reveals that for a couple stress fluid, there exist two stagnation points; for example, at $\mathrm{t}=0$, one of them lies between $\mathrm{X}=0.25$ and $\mathrm{X}=0.5$, and the other between $\mathrm{X}=0.5$ and $\mathrm{X}=0.75$. A similar observation was reported earlier for a similar study [53]. Figs 3.6-3.7 reveal that for free pumping case $(\Delta p=0)$, the magnitude of the velocity for Newtonian as well as couple stress fluid in the forward and retrograde flow regions increases when the value of the amplitude ratio increases. Moreover, from Fig 3.7, one may observe that as the value of $\alpha$ increases, the velocity in the forward flow region increases. It may also be noted that the couple stress parameter $\alpha$ enhances the flow velocity in the retrograde flow region. The influence of the Darcy number Da on the velocity profile for a couple stress fluid (with $\alpha=0.1$ ) is depicted in Fig. 3.8. One can further note that as the Darcy number Da decreases, the axial velocity diminishes in forward region, whereas it increases in magnitude in retrograde flow region (i.e retrograde flow enhances). It is worthwhile to mention here that the parabolic nature of the velocity profile is disturbed in the central region for small values of Da. We further observe that for a couple stress fluid, the axial velocity decreases with an increase in the value of the 


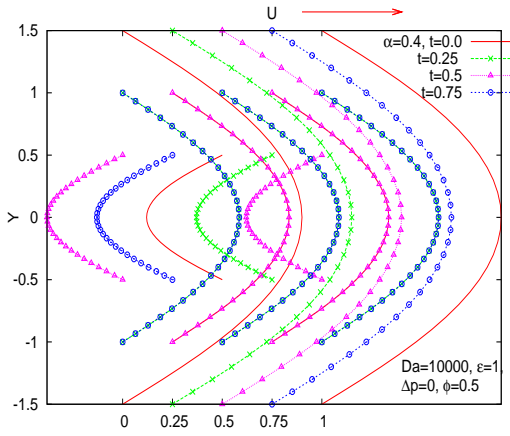

Fig. 3.5

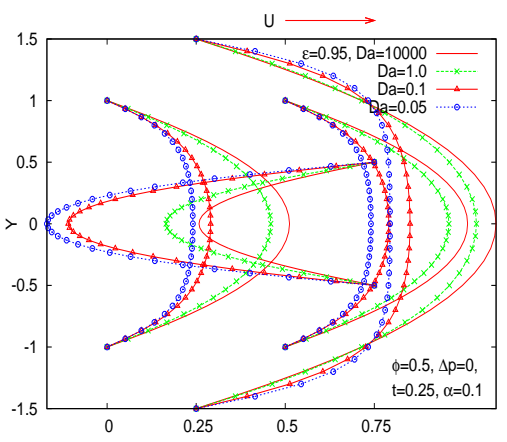

Fig. 3.8

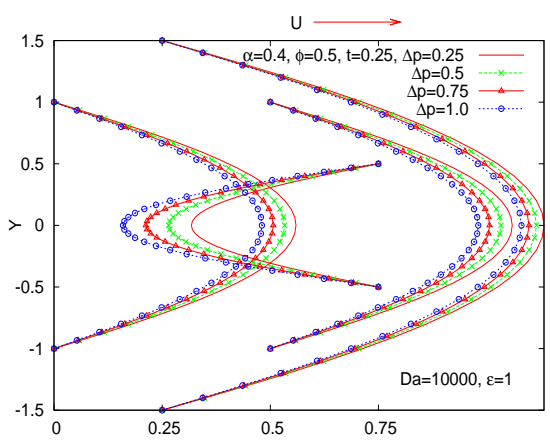

Fig. 3.11

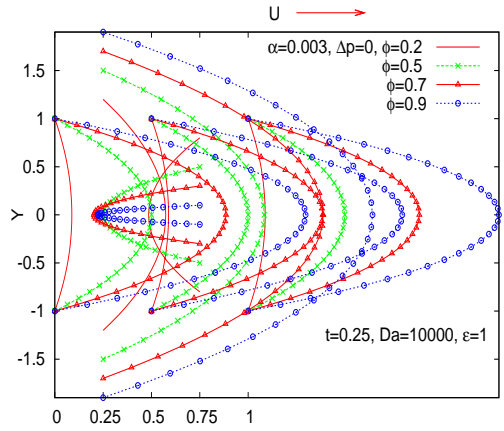

Fig. 3.6

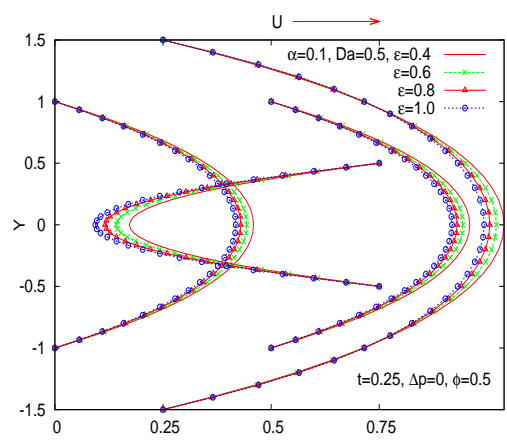

Fig. 3.9

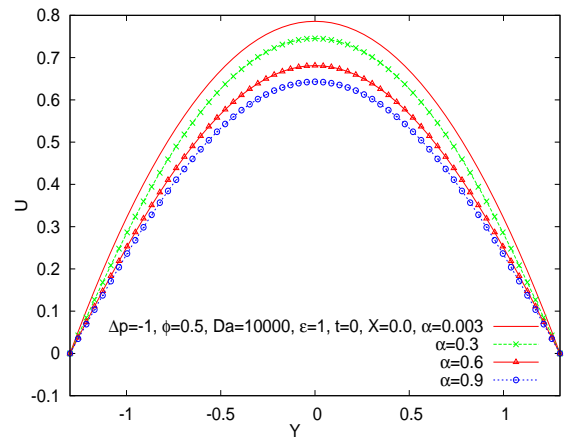

Fig. 3.12

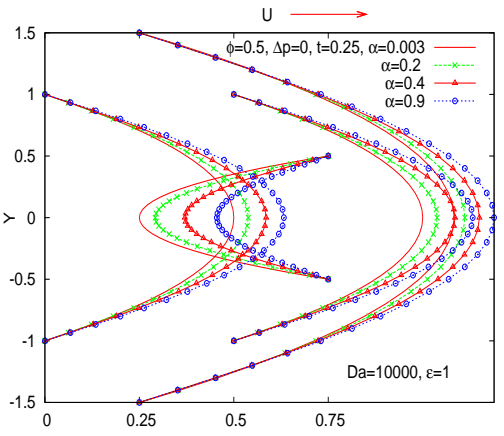

Fig. 3.7

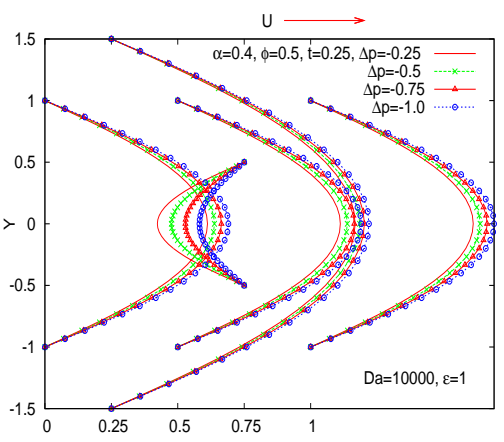

Fig. 3.10

Figs. 3.5-3.12: Longitudinal velocity profiles in different situations 
porosity parameter $\epsilon$ of the channel (cf. Fig3.9) in both forward and backward flow regions. Figs. 3.10-3.11 give the distribution of velocity of a couple stress fluid for co-pumping $(\Delta p<0)$ and pumping $(\Delta p>0)$ cases. From both the figures, it is revealed that as the value of $\Delta p$ decreases, the velocity increases in both the flow regions. It is important to mention that unlike free pumping case, in the co-pumping case with $\Delta p=-1$, the velocity reduces with the increase in the value of the couple stress parameter $\alpha$ (cf. Fig. 3.12).

\subsection{Frictional Force}

The average rise in frictional force in the case of a couple stress fluid are calculated over one wave period. Figs. 4.(a) depict the variation of frictional force with time for different values of $\bar{Q}$ when $\phi=0.5$. It can be observed that the effect of increasing the flow rate $\bar{Q}$ is to enhance the frictional force F. As expected, from Figs. 4(b), we find that F decreases with rise in amplitude ratio. Figs. 4(c) reveal that the magnitude of the frictional force for a Newtonian fluid is less than that in the case of a couple stress fluid and that as $\alpha$ increases, the magnitude of $\mathrm{F}$ enhances. The quantum of influence of the Darcy number on frictional force is shown in Figs. 4(d). These figures indicate that the magnitude of $\mathrm{F}$ diminishes when the value of Da reduces, while Figs. 4(e) show that with an increase in the porosity parameter $\epsilon$, the magnitude of the frictional force decreases.

\subsection{Streamline Patterns and Trapping}

Under certain conditions the streamlines on the center line in the wave frame of reference are found to split in order to enclose a bolus of fluid particles circulating along closed streamlines. This phenomenon is referred to as trapping, which is a characteristic of peristaltic motion. Since this bolus appears to be trapped by the wave, the bolus moves with the same speed as that of the wave. Figs. 5.1 illustrate the streamline patterns and trapping for different values of the couple stress parameter $\alpha$ corresponding to $\bar{Q}=1.3, \phi=0.3, D a=10000, \epsilon=1$. It is important to observe that the size of the trapped bolus increases as the couple stress parameter $\alpha$ increases. Formation of trapping zone and streamline patterns is depicted in Figs. 5.2 for different values of Darcy number Da, when $\alpha=0.003, \epsilon=0.8, \phi=0.5, \bar{Q}=1.4$. These figures reveal that with a reduction in Da, the trapping zone decreases and it disappears completely when Da attains the value 0.08. Plots showing the effect of the porosity parameter $\epsilon$ on trapping are presented in Figs. 5.3. One can observe that size of the trapped bolus decreases with increase in $\epsilon$ and vanish 


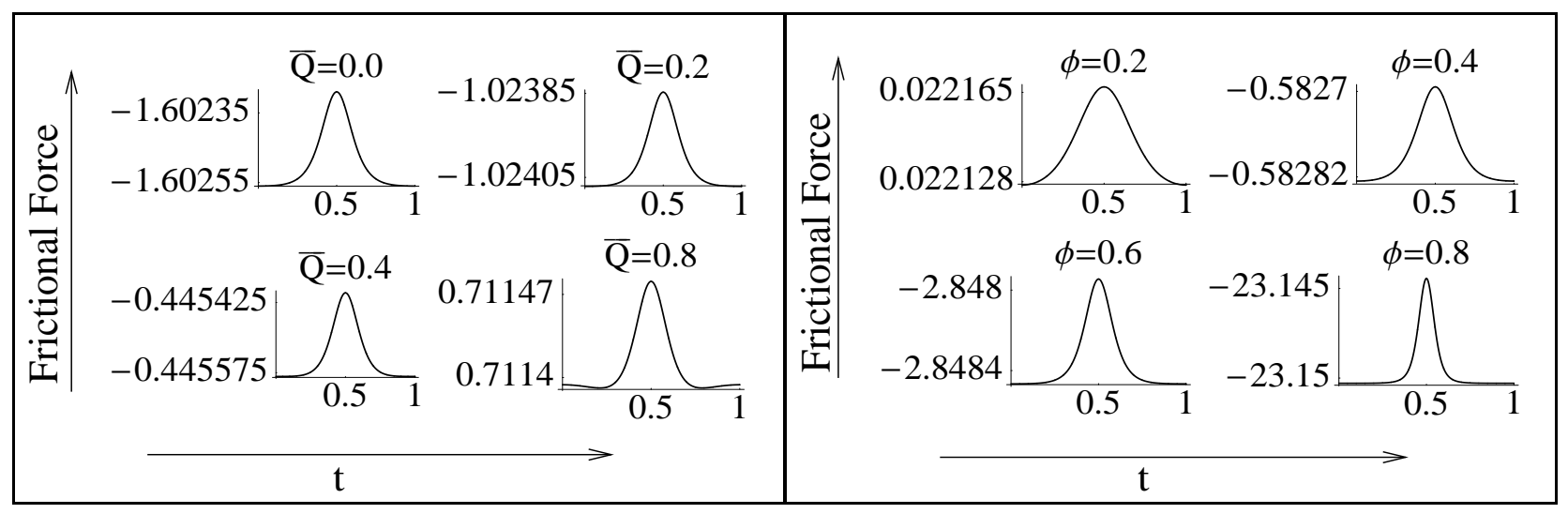

(a)

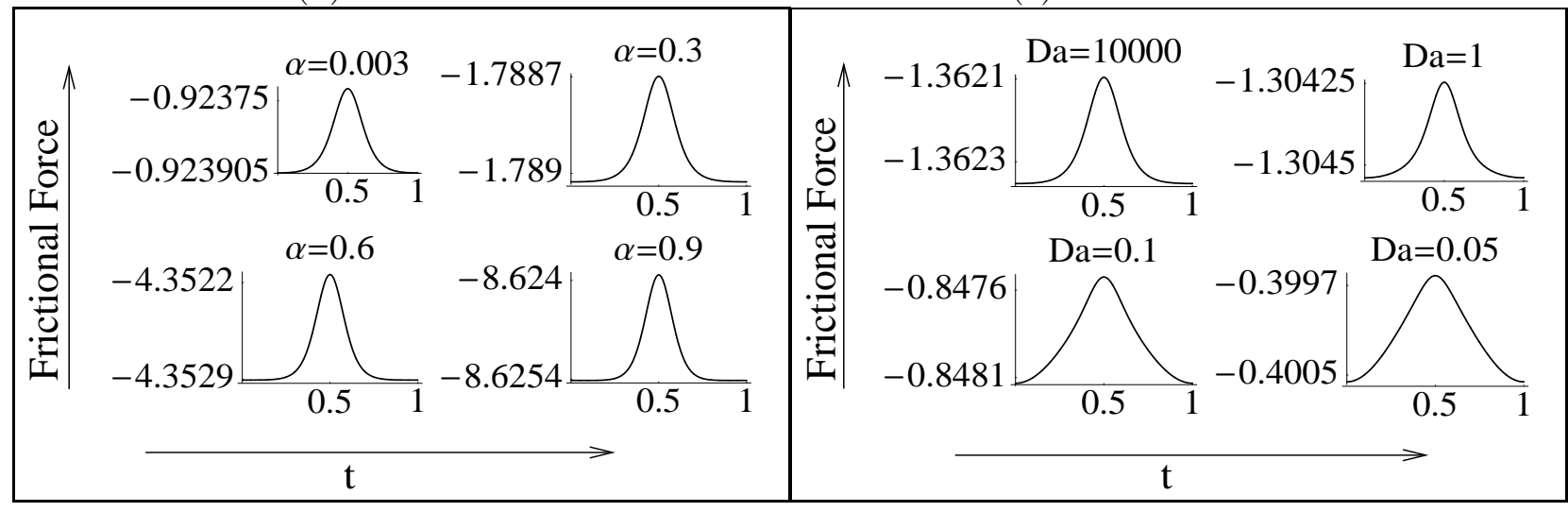

(c)

$(d)$

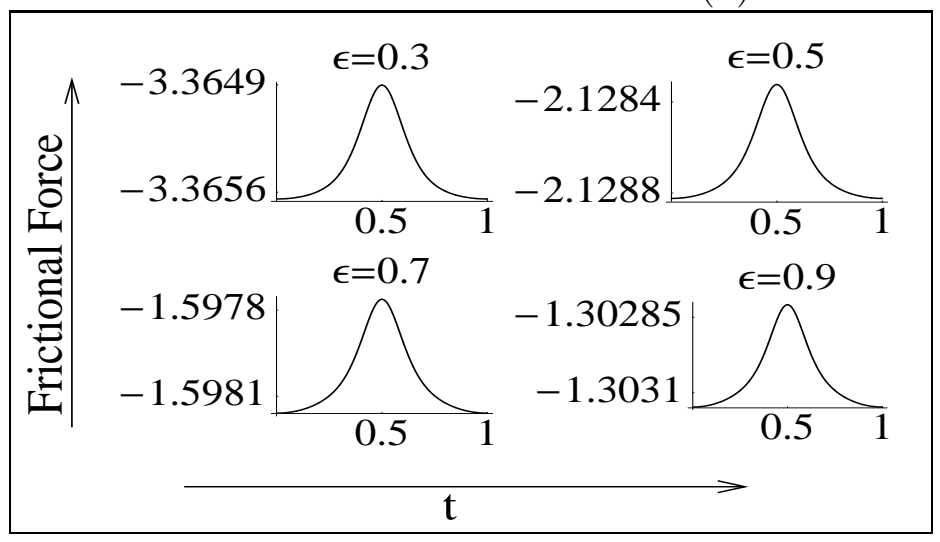

(e)

Figs. 4: Frictional force versus time for different cases

(a) $\alpha=0.2, \phi=0.5, D a=10000, \epsilon=1$

(b) $\alpha=0.2, \bar{Q}=0.1, D a=10000, \epsilon=1$

(c) $\phi=0.5, \bar{Q}=0.1, D a=10000, \epsilon=1$

(d) $\alpha=0.2, \phi=0.5, \bar{Q}=0.1, \epsilon=0.95$

(e) $\alpha=0.2, \phi=0.5, \bar{Q}=0.1, D a=0.5$ 

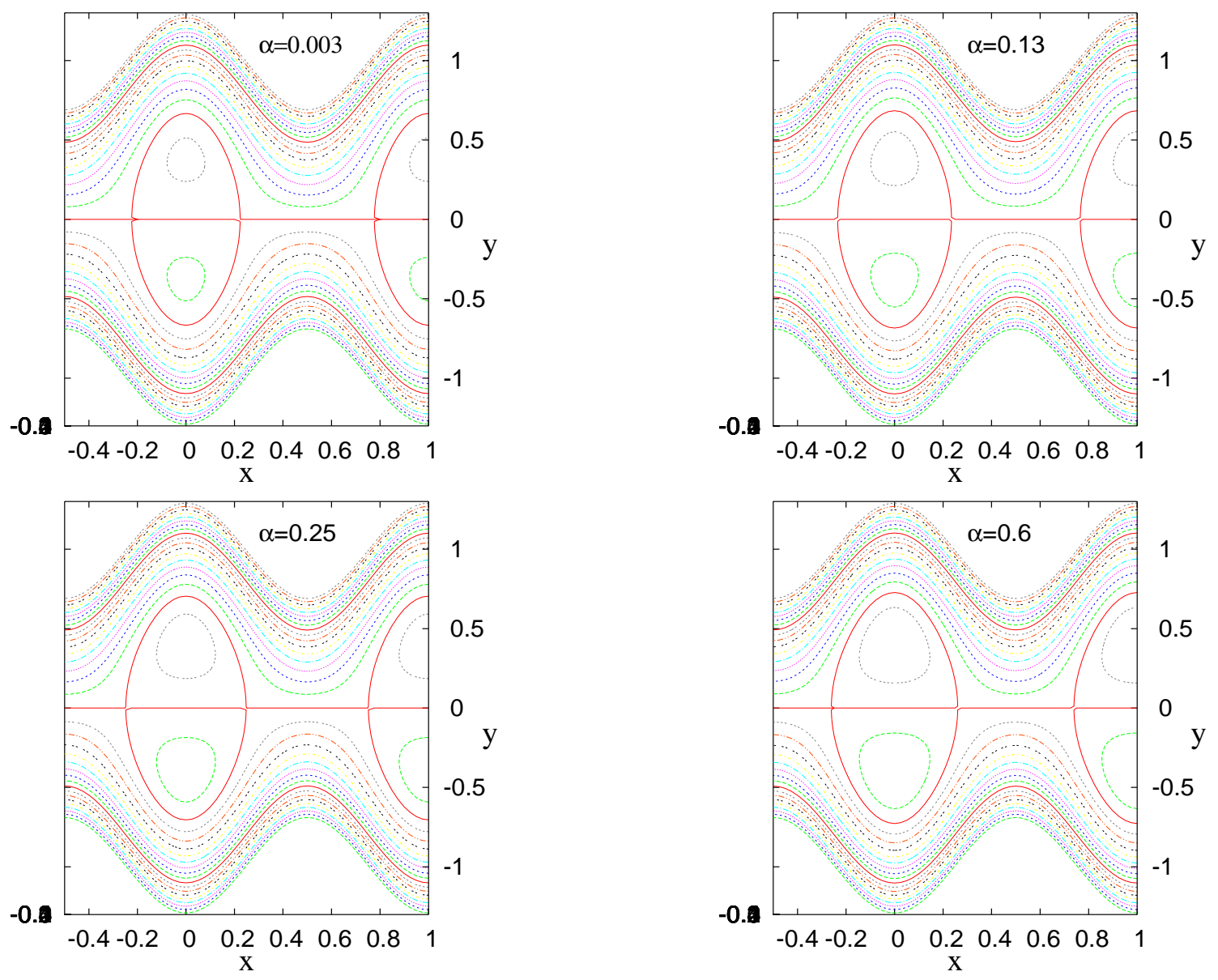

Figs 5.1: Streamline patterns for different values of $\alpha$ with $\bar{Q}=1.3, \phi=0.3, D a=10000, \epsilon=1$ 

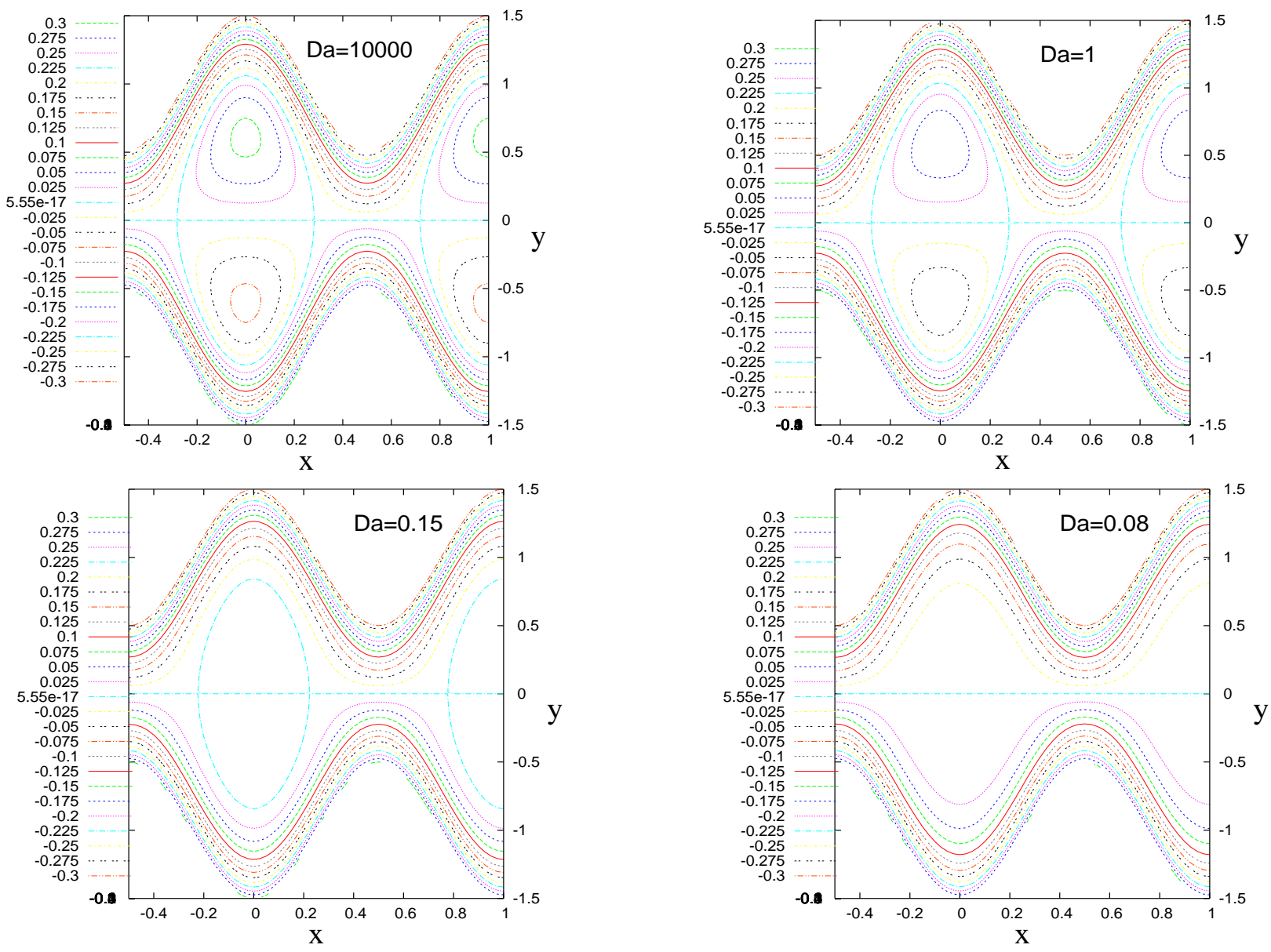

Figs. 5.2: Streamline patterns for different values of Da with $\alpha=0.003, \bar{Q}=1.4, \phi=0.5, \epsilon=$ 0.8

when $\epsilon=1$. Figs. 5.4 indicate that size of trapped bolus increases as the flow rate increases and that with increasing flow rate, the bolus gets shifted towards the boundary more and more.

\section{Summary and Conclusion}

Peristaltic transport of a couple stress fluid in a porous medium has been the concern of the present investigation. The study particularly pertains to a situation when the Reynolds number is low and curvature of the channel is quite small. The primary motivation behind the study has been to have an insight into the physiological problem of peristaltic transport of blood in a segment of the micro-circulatory system, when the segment is in a pathological state, whereby the lumen of the particular segment turns into a porous structure. Emphasis has been paid to investigate the peristaltic pumping characteristics, blood velocity, frictional force, streamline 

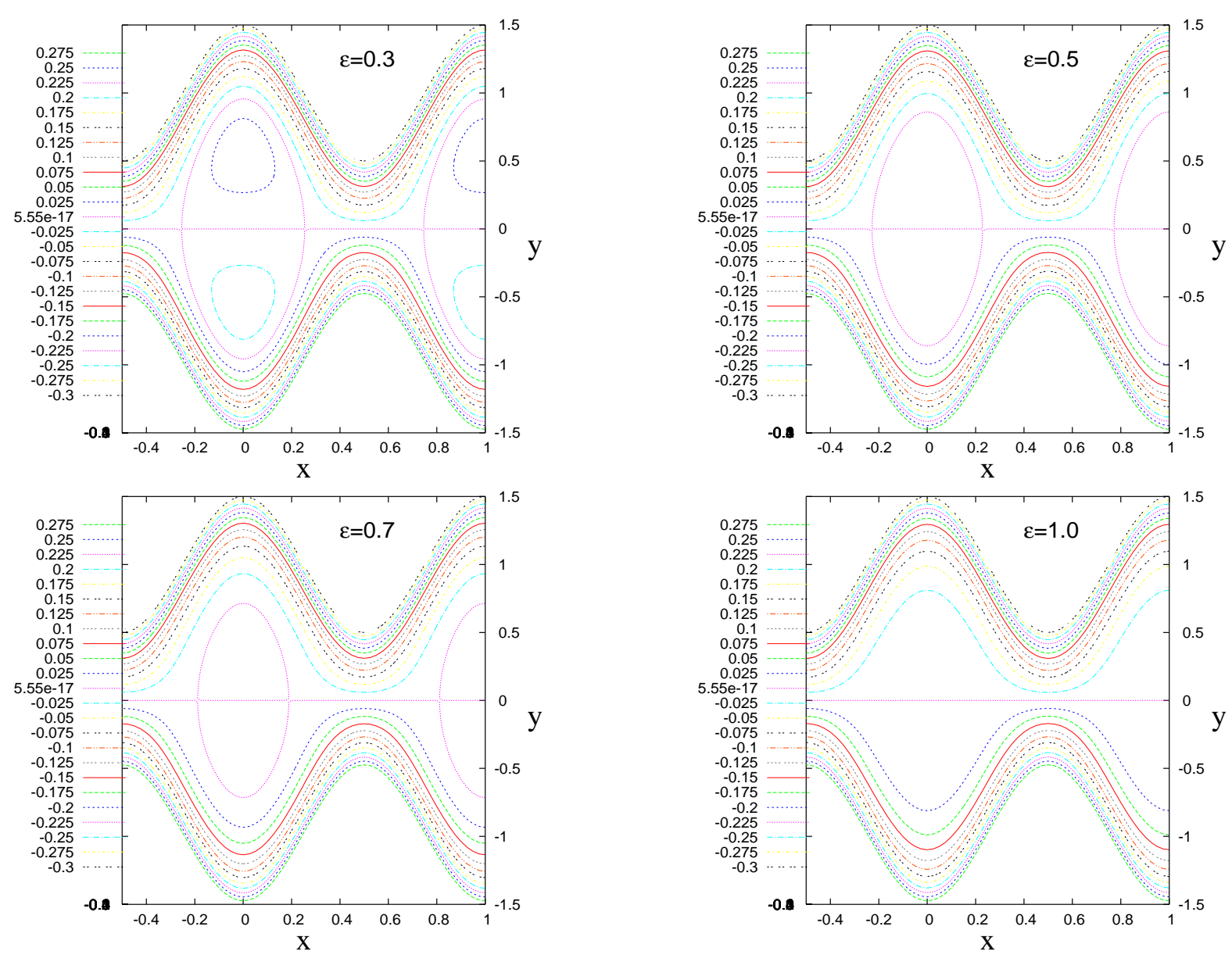

Figs. 5.3: Streamline patterns for different values of $\epsilon$ with $\alpha=0.003, \bar{Q}=1.4, \phi=0.5, D a=$ 0.1 

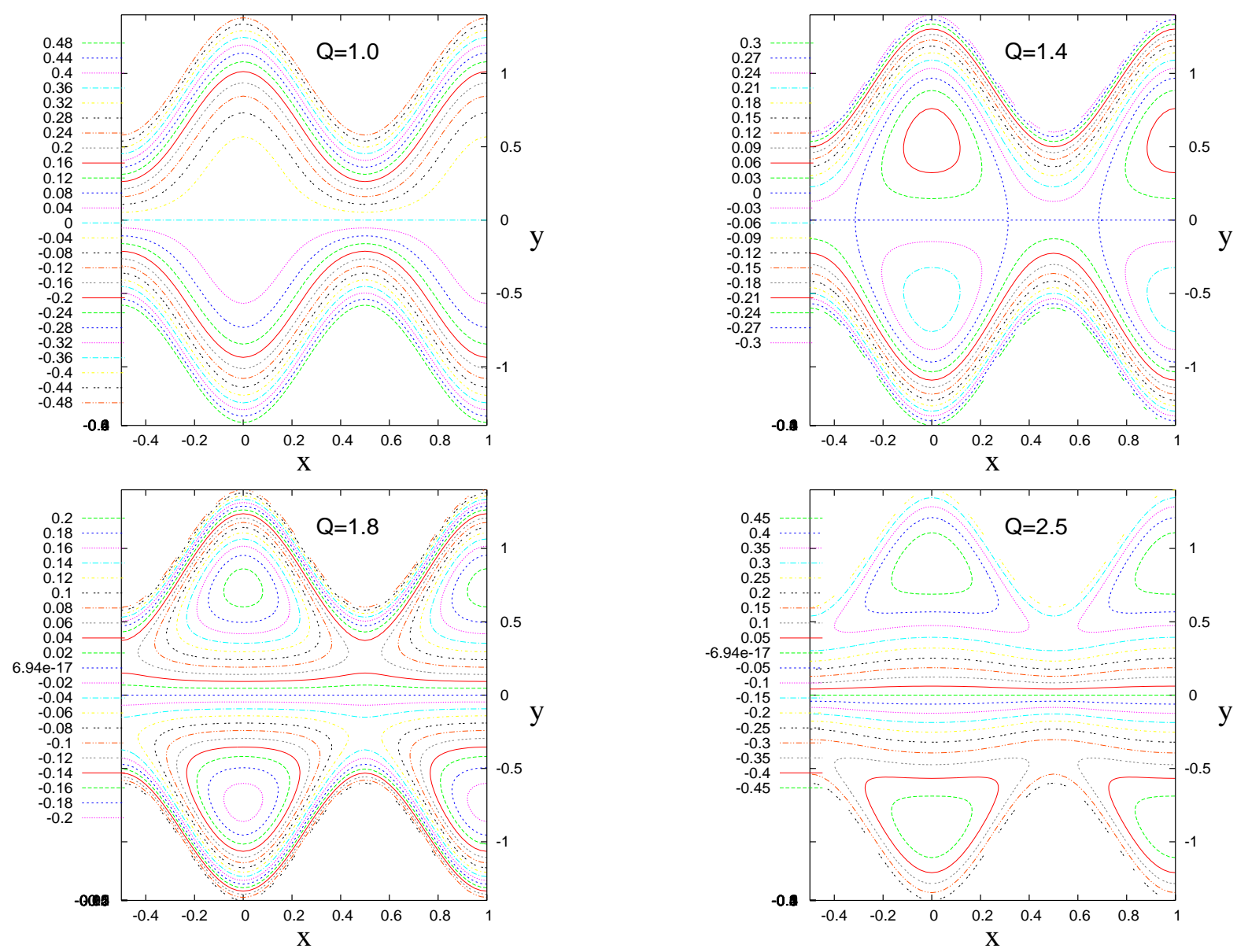

Figs. 5.4: Streamline patterns for different values of $\bar{Q}$ with $\alpha=0.003, \phi=0.4, D a=$ 10000, $\epsilon=1$ 
patterns and trapping as a function of the peristaltic wave amplitude as well as Darcy permeability and porosity parameters. Although the analysis starts with an analytical procedure, suitable numerical techniques have been employed whenever analytical methods have been found inadequate. The results and observations of this study (reduced to some particular cases) are in good agreement with those reported earlier by other investigators (cf. [52]). A very significant conclusion that can be made on the basis of this study is that it is possible to enhance the pressure rise $\Delta p$ as well as the peristaltic pumping performance by increasing the amplitude ratio/couple stress parameter and/or by reducing the porosity. The study further reveals the important fact that change in velocity is strongly affected by the wave amplitude, the couple stress effect and the porosity factor. The other important conclusion that can be drawn out of this study is that while flow reversal is enhanced due to increase in pressure rise in the free pumping case, it is possible to bring about reduction in flow reversal by increasing the couple stress effect/permeability. Moreover, the occurrence of trapping can be reduced/eliminated by decreasing the permeability of the medium through the application of some appropriate mechanism. On the basis of this study we can make an important conjecture that when the pressure rise $\Delta p$ is negative, the couple stress effect creates resistance to flow, whereby the velocity is reduced; however, the flow is enhanced due to the couple stress effect in the case of free pumping $\Delta p=0$

The present study reveals that it is possible to increase both pumping and pressure by increasing the amplitude ratio and couple stress parameter and also by reducing the permeability (Darcy number). The present study reveals further that the velocity change is strongly dependent on $\phi, \alpha$ and porosity. Lastly, the magnitudes of $\Delta p, \phi$ and $\alpha$ affect significantly the nature of flow reversal.

\section{Acknowledgments}

The authors are thankful to the reviewers for their kind words of profound appreciation in respect of the quality and presentation of the work. One of the authors (Somnath Maiti) is thankful to the Council of Scientific and Industrial Research (CSIR), New Delhi for awarding him a senior research fellowship. 


\section{References}

[1] Srivastava LM, Srivastava VP, Peristaltic transport of blood: Casson model : II, J Biomech 17(11):821-829, 1984.

[2] Eytan O, Jaffa AJ, Elad D, Peristaltic flow in a tapered channel: application to embryo transport within the uterine cavity, Med Engng Phy 23:473-82, 2001.

[3] Jimenez-Lozano J, Sen M, Dunn PF, Particle motion in unsteady two-dimensional peristaltic flow with application to the ureter, Phys Rev E 79: 041901, 2009.

[4] Misra JC, Pandey SK, Peristaltic transport of a non-Newtonian fluid with a peripheral layer, Int J Eng Sci 37:1841-1858, 1999.

[5] Misra JC, Pandey SK, Peristaltic flow of a multi-layered power-law fluid through a cylindrical tube, Int J Eng Sci 39:387-402, 2001.

[6] Misra JC, Pandey SK, Peristaltic transport of blood in small vessels: study of a mathematical model, Comput Math Appl 43:1183-1193, 2002.

[7] Misra JC, Pandey SK, Peristaltic transport of a particle-fluid suspension in a cylindrical tube, Comput Math Appl 28:131-145, 1994.

[8] Misra JC, Pandey SK, Peristaltic transport in a tapered tube", Math. Comput. Model 22:137$151,1995$.

[9] Misra JC, Pandey SK, A mathematical model for oesophegeal swallowing of a food-bolus, Math Comput Model 33:997-1009, 2001.

[10] Misra JC, Maiti S, Shit GC, Peristaltic Transport of a Physiological Fluid in an Asymmetric Porous Channel in the Presence of an External Magnetic Field, J Mech Med Biol 8(4):507$525,2008$.

[11] Misra JC, Pandey SK, Peristaltic transport of physiological fluids, In Misra JC, Biomathematics: Modelling and Simulation, 166-193, World Scientific, London/Singapore, 2006.

[12] Maiti S, Misra JC, Peristaltic Flow of a Fluid in a Porous Channel: a Study Having Relevance to Flow of Bile, Int J Eng Sci 49:950-966, 2011. 
[13] Usha S, Rao AR, Effect of curvature and inertia on the peristaltic transport in a two fluid system, Int J Engng Sci 38: 1355-1375, 2000.

[14] Mishra M, Rao AR, Peristaltic transport of a Newtonian fluid in an asymmetric channel, ZAMP 54:532-550, 2003 .

[15] Rao AR, Mishra M, Peristaltic transport of a power law fluid in a porous tube, J NonNewtonian Fluid Mech 121:163-174, 2004.

[16] Park CY, Shur M, Dewey CF, A Study of fluid-membrane interactions that limit the output of peristaltic micropumps, J Mech Med Biol 11(2):325-326, 2011.

[17] Akbar, NS, Nadeem S, Analytical and numerical solutions of peristaltic flow of Williamson fluid model in an endoscope, J Mech Med Biol 0(1):1-16, 2010.

[18] Antanovskii LK, Ramkissoon H, Long-wave peristaltic transport of a compressible viscous fluid in a finite pipe subject to a time-dependent pressure drop, Fluid Dyna Res 19:115-123, 1997.

[19] Bergel DH, Cardiovascular Fluid Dynamics, Academic Press, London, 1972.

[20] Dash RK, Mehta KN, Jayaraman G, Casson fluid flow in a pipe filled with a homogeneous porous medium, Int J Eng Sci 34(10):1145-1156, 1996.

[21] Misra JC, Chakravarty S, Flow in arteries in the presence of stenosis, J Biomech 19:907-916, 1986.

[22] Misra JC, Patra MK, Misra SC, A Non-Newtonian Fluid Model for Blood Flow Through Arteries Under Stenotic Conditions, J Biomech 26:1129-1141, 1993.

[23] Misra JC, Shit GC, Flow of a Biomagnetic Visco-Elastic Fluid in a Channel With Stretching Walls, J App Mech (ASME) 76(6):061006, 2009.

[24] Misra JC, Shit GC, Role of slip velocity in Blood flow through stonosed arteries: a nonNewtonian model, J Mech Med Biol 7:337-353, 2007.

[25] Misra JC, Sinha A, Shit GC, Magnetohydrodynamic flow of blood in a porous channel, $J$ Mech Med Biol (in press), 2011. 
[26] Misra JC, Adhikary SD, Shit GC, Multiphase flow of blood through arteries with a branch capillary: a theoretical study, J Mech Med Biol 7:395-417, 2007.

[27] Misra JC, Sinha A, Shit GC, Theoretical analysis of blood flow through an arterial segment having multiple stenoses, J Mech Med Biol 8:265-279, 2008.

[28] Chien S, Usami S, Jan KM, Skalak R, Haemorheological Models, in Rheology of Biological Systems, Ed. H. L. Gabelnick and M. Litt, 12-48, Illinois, USA, 1973.

[29] Beg OA, Giants of Engineering Science, Chapter 2: Richard Skalak and Biofluid Engineering Science, Matador, Leicester, UK (2003) (translated into Chinese, 2008).

[30] Charm SE, Kurland GS, Viscometry of human blood for shear rates of 0-100,000 $\mathrm{sec}^{-1}$, Nature 206:617-618, 1965.

[31] Charm SE, Kurland GS, Blood Flow and Microcirculation, John Wiley, New York, pp. 36, 1974.

[32] Blair GWS, Spanner DC, An Introduction to Bioreheology, Elsevier, Amsterdam, 1974.

[33] Cowin SC, The theory of polar fluids. Advances in Applied Mechanics, Editor: C.S. Yih, 14, Academic Press, New York, pp. 279-347, 1974.

[34] Beg OA, Prasad VR, Vasu B, Reddy NB, Li Q, Bhargava R, Free convection heat and mass transfer from an isothermal sphere to a micropolar regime with Soret/Dufour effects, Int $J$ Heat Mass Trans 54:9-18, 2011.

[35] Beg OA, Zueco J, Chang TB, Numerical analysis of hydromagnetic gravity-driven thin film micropolar flow along an inclined plane, Chemical Eng Communications 198(3):312-331, 2011.

[36] Srivastava LM, Peristaltic transport of a couple-stress fluid, Rheol Acta 25:638-641, 1986.

[37] Zueco J, Beg OA, Network numerical simulation applied to pulsatile non-Newtonian flow through a channel with couple stress and wall mass flux effects, Int J Appl Math Mech 5(2):1-16, 2009.

[38] Ghosh SK, Beg OA, Bhargava R, Rawat S, Beg TA, Mathematical modelling of transient magnetohydrodynamic couple stress fluid flow in a rotating channel, Int J Appl Math Mech 6(6):23-45, 2010. 
[39] Valanis KC, Sun CT, Poiseuille Flow of Fluid with Couple Stress with Applications to Blood Flow, Biorheology 6(2):85-97, 1969.

[40] Popel AS, Regirer SA, Usick PI, A Continuum Model of Blood Flow,, Biorheology 11:427$437,1974$.

[41] Stokes VK, Couple Stresses in Fluids, Phys Fluids 9:1709-1715, 1966.

[42] Shehawey EFE, Mekheimer KS, Couple stresses in peristaltic transport of fluids, J Phys D: Appl Phys 27:1163-1170, 1994.

[43] Rashidi MM, Keimanesh M, Beg OA, Hung TK, Magneto-hydrodynamic biorheological transport phenomena in a porous medium: A simulation of magnetic blood flow control and filtration, Int J Numer Meth Biomed Engn 27:805-821, 2011.

[44] Zueco J, Beg OA, Beg TA, Numerical solutions for unsteady rotating high-porosity medium channel Couette hydrodynamics, Physica Scripta 80:1-8, 2009.

[45] Beg OA, Zueco J, Takhar HS, Unsteady magnetohydrodynamic Hartmann-Couette flow and heat transfer in a Darcian channel with Hall current, ionslip, viscous and Joule heating effects: Network numerical solutions, Commu Nonlinear Sci Numer Simulation 14:10821097, 2009.

[46] Beg OA, Bhargava R, Rawat S, Takhar HS, Halim MK, Computational modeling of biomagnetic micropolar blood flow and heat transfer in a two-dimensional non-Darcian porous medium, Meccanica 43(4):391-410, 2008.

[47] Beg OA, Takhar HS, Bhargava R, Rawat S, Prasad VR, Numerical study of heat transfer of a third grade viscoelastic fluid in non-Darcian porous media with thermophysical effects, Physica Scripta 77:1-11, 2008.

[48] Zueco J, Beg OA, Ghosh SK, Unsteady hydromagnetic natural convection of a shortmemory viscoelastic fluid in a non-Darcian regime: network simulation, Chemical Engn Communications 198:172-190, 2011.

[49] Beg OA, Zueco J, Lopez-Ochoa LM, Non-linear transient hydromagnetic partially ionised dissipative Couette flow in a non-Darcian porous medium channel with Hall, ionslip and Joule heating effects, Progress Comput Fluid Dyn 11(2):116-129, 2011. 
[50] Beg OA, Zueco J, Ghosh SK, Heidari A, Unsteady magnetohydrodynamic heat transfer in a semi-infinite porous medium with thermal radiation flux: analytical and numerical study, Advances Numer Analy 2011:1-17, 2011.

[51] Bhargava R, Sharma S, Takhar HS, Beg TA, Beg OA, Hung TK, Peristaltic pumping of micropolar fluid in porous channel : model for stenosed arteries, J Biomech 39(Supplement 1):S649, 2006.

[52] Shapiro AH, Jaffrin MY, Weinberg SL, Peristaltic pumping with long wavelength at low Reynolds number, J Fluid Mech 37:799-825, 1969.

[53] Takabatake S, Ayukawa K, Numerical study of two-dimensional peristaltic flows, J Fluid Mech 122:439-465, 1982.

[54] Takabatake S, Ayukawa K, Mori A, Peristaltic pumping in circular cylindrical tubes: a numerical study of fluid transport and its efficiency, J Fluid Mech 193:267-283, 1988.

[55] Sugihara-Seki M, Fu BM, Blood ow and permeability in microvessels" Fluid Dyn Res 37:82132, 2005.

[56] Guyton AC, Hall JE, Text Book of Medical Physiology, Elsevier: Saunders Co, Philadelphia (USA), 2006.

[57] Nield DA, Modelling fluid flow in saturated porous media and at interface, In: Ingham D.B and Pop I.(Eds.) Transport phenomena in porous media II Pergamon 1-19, 2002

[58] Barbee KA, Davies PF, Lal R, Shear stress-induced reorganization of the surface topography of living endothelial cells imaged by atomic force microscopy, Circ Res 74:163-171, 1994.

[59] Singh C, Lubrication theory for couple stress fluids and its applications to short bearing, Wear 80:281-290, 1982. 